\title{
How We Approach: Severe Congenital Neutropenia and Myelofibrosis due to Mutations in VPS45
}

\author{
Bella Shadur ${ }^{1,2,3}$, Nathalie Asherie ${ }^{1}$, Peter E. Newburger ${ }^{4}$, and Polina Stepensky ${ }^{1}$ \\ 1.Bone Marrow Transplantation Department, Hadassah-Hebrew University Medical Center, \\ Jerusalem, Israel \\ 2.Garvan Institute of Medical Research, Sydney, Australia \\ 3.University of New South Wales, Sydney, Australia \\ 4.Departments of Pediatrics \& Molecular, Cell, and Cancer Biology, University of Massachusetts \\ Medical School, Worcester, Massachusetts, USA
}

\begin{abstract}
Mutations in the VPS45 gene lead to a severe primary immune deficiency characterised by severe congenital neutropenia and primary myelofibrosis, leading to overwhelming infection and early death. This condition is exceedingly rare with only 16 patients previously reported, including four with successful hematopoietic stem cell transplantation. We review the pathophysiology underlying this condition and detail our approach to treatment, particularly vis-à-vis bone marrow transplantation and the challenges of transplanting into a diseases bone marrow niche. We provide an update on the progress of our three previously reported patients, and two additional patients transplanted at our center.
\end{abstract}

\section{Keywords}

Myelofibrosis; severe combined neutropenia; infection; bone marrow transplantation

\section{Introduction}

VPS45 deficiency is a hereditary disease characterised by severe congenital neutropenia $(\mathrm{SCN})$ with bacterial and fungal infections, primary myelofibrosis (PMF) with extramedullary hematopoiesis including nephromegaly, bone marrow failure, defective cell migration, possible neurological impairment, and early death without hematopoietic stem cell transplantation (HSCT) ${ }^{3-6}$ Though exceptionally rare, we believe VPS45 offers instructive learning points at the intersection of primary immune deficiency, hematopoiesis, and transplant biology. ${ }^{3,7}$ In this paper, we detail our experience in treating five pediatric

Compliance with Ethical Standards

Conflicts of interest The authors declare that they have no conflict of interest.

Informed consent Not applicable 
patients with inherited mutations in the VPS45 gene and make more general recommendations regarding management, particularly with reference to HSCT.

The VPS45 gene is located at chromosome 1q21-q22 and functionally relevant mutations of this protein were first described in humans in 2013. ${ }^{4,5}$ All reported patients have demonstrated homozygous inheritance and three distinct mutations have previously been published: c. $671 \mathrm{C}>\mathrm{A}$; p.T224A ${ }^{4,5,7}$, c. $712 \mathrm{G}>\mathrm{A}$; p.E238K ${ }^{3}$, and c. $1403 \mathrm{C}>\mathrm{T}$; p.P468L ${ }^{6}$. One additional homozygous mutation (Lisa Madden, personal communication) c.1157G>A; p. R386H occurred in a patient of Mexican and Mexican-Irish descent, with no known consanguinity. Although these mutations are not located near each other in the VPS45 gene sequence, three-dimensional modelling predicts that their encoded amino acids are all located in the key hinge region of the protein). ${ }^{6}$ Thus, VPS45 mutations lead to a deficient and defective protein, and can be viewed through the prism of three distinct but interconnected categories of disease: defective endosomal-lysosomal protein trafficking, SCN, and PMF.

VPS45 is a member of the Sec1p/Munc18-like family of proteins that are vital for endosomal/lysosomal protein trafficking. ${ }^{8,9}$ VPS45 performs its task through interaction with SNAREs (Soluble N-ethylmaleimide-sensitive factor Attachment protein Receptors) which mediate membrane fusion. ${ }^{10}$ Studies suggest that defects in the amount or function of VPS45 causes abnormal SNARE binding, ${ }^{9}$ leading to impaired degranulation, release of inflammatory mediators, and impaired migration due to decreased levels of B1-integrin and SNARE syntaxin-16. ${ }^{11}$

Migration, endocytosis, and degranulation are key functions of neutrophils, which are severely affected in both number and function in this disorder. ${ }^{4,12}$ As such, VPS45 is sometimes referred to as severe congenital neutropenia Type 5 (SCN5). ${ }^{6}$ Whilst neutrophil granules can be formed, they cannot be released from the cell because of impaired trafficking of endosomes, and the neutrophils themselves demonstrate an impaired ability to migrate to areas of infection and inflammation. This form of SCN does not respond to treatment with granulocyte-colony stimulating factor (G-CSF) which may occur secondary to myelofibrosis, intrinsic neutrophil defects, or both. ${ }^{4,5}$ Similar to other forms of SCN (e.g. due to mutations in ELANE, G6PC3), the neutrophils of VPS45 also demonstrate increased apoptosis. ${ }^{4,5,12,13}$

Finally, VPS45 deficiency causes PMF, an exceptionally rare entity in children. Unlike adult-onset PMF, it is not associated with malignant transformation but rather features hypercellularity, megakaryocyte hyperplasia, moderate-to-severe reticulin fibrosis and collagen fibrosis, and progressive bone marrow failure with extramedullary hematopoiesis. The exact mechanism underlying this phenomenon is not known, although increased release of transforming growth factor (TGF)- $\beta$ and other mediators from apoptotic neutrophils and abnormal megakaryocytes may play a role. Platelets of patients with VPS45 deficiency have shown a decrease in the content of a-granules. These lysosome-like organelles involved in the endosomal-lysosomal pathway of platelets contain pro-fibrotic factors such as TGF- $\beta$ and platelet-derived growth factor; lack of these granules is associated not only with 
thrombocytopenia and thrombasthenia as in VPS45-deficient patients, but also myelofibrosis, as in grey platelet syndrome. ${ }^{3-7,14}$

\section{Clinical Features}

To date, 16 patients with VPS45 mutations have been reported in the literature. Common clinical features that should warrant consideration of a diagnosis of VPS45 deficiency include profound neutropenia in the first months of life that fails to respond to G-CSF therapy, failure to thrive, infections consistent with neutropenia, and myelofibrosis with bone marrow failure leading to extramedullary hematopoiesis with hepatosplenomegaly and the unique feature of nephromegaly. VPS45 defect carries an exceptionally poor prognosis with eight of the 16 reported patients dying before, during, or after hematopoietic stem cell transplantation. Death occurs early, with eight patients dying between three months and three years of age. All reported patients have been born to consanguineous parents, and the disease has so far been reported in three ethnic groups: Palestinian $(n=12)$, Moroccan $(n=$ 3), and Mexican $(n=1)$. All Palestinian patients treated thus far harbor the c.671 C>A; p.T224A mutation whereas all three Moroccan patients inherited biallelic c.712 G>A; p.E238K mutations; the Mexican patient was found to have homozygous c.1403 C>T; p.P468L mutations. ${ }^{3-7}$

Whilst most patients do not demonstrate neurological findings, all three Moroccan patients with the c.712 G>A; p.E238K mutation demonstrated neuromotor delay. The patient reported by Meerschaut et al demonstrated delayed ability to sit and stand without support, generalized hypotonia, nystagmus, cortical blindness and spoke her first words at 40 months of age. She also had unusual facial features not noted in other patients: rounded facies, a prominent forehead, long almond-shaped palpebral fissures, bulbous nasal tip with a short columella, and a thin upper lip, as well as bilateral fifth finger clinodactyly. ${ }^{3}$ The additional Moroccan children, siblings described by Vilboux et al, demonstrated cortical blindness, hearing loss, thin corpus callosum on magnetic resonance imaging, and dysrhythmia on electroencephalography. ${ }^{4}$ No patients with the c.671 C>A; p.T224A mutation demonstrated such changes, although one patient reported by Stepensky et al developed a pervasive developmental disorder with behavioural regression four months after HSCT; magnetic resonance imaging and neurological examination were normal. ${ }^{5,7}$ The Mexican patient with c.1403 C > T; p.P468L mutations reported by Shah et al demonstrated nystagmus and ataxia with cerebellitis. ${ }^{6}$ Given the consanguineous parentage of all these children, it is possible that additional homozygous mutations were responsible for these neurological changes; however genetic investigations did not find mutations in any known genes. ${ }^{3-7}$

Bone marrow aspiration has invariably led to a dry tap, and bone marrow biopsy specimens showed fibrosis with increased reticulin staining. The patient described by Meerschaut et al was noted to have delayed separation of her umbilical cord at five weeks of age and only 11 ribs; ${ }^{3}$ these findings were not noted in any other reported cases. Despite the severe lymphopenia that can develop, only one patient developed infection with suspected Pneumocystis jirovecii. ${ }^{6}$ 


\section{HSCT: Indications and Management}

Patients with VPS45 gene mutations can be supported for some time with antimicrobial therapy and regular transfusion of blood and platelets; HSCT, however, offers the only hope of cure for this disease. Seven cases of HSCT for VPS45 protein deficiency have been reported in the literature, with four patients surviving the procedure and three dying posttransplant (Table 1). All non-surviving patients demonstrated engraftment failure. Vilboux et al do not detail the transplant protocol used in their two deceased patients ${ }^{4}$ (Patient 2 and 3) but we used an umbilical cord blood graft with fludarabine, treosulphan, thiotepa and antithymocyte globulin (ATG); the patient (Patient 4) died on day +31 following transplantation. 5,7

Meerschaut et al successfully transplanted their patient (Patient 6) at 18 months of age, despite extensive neurological deficits, using a 9/10 matched unrelated donor and a chemotherapy regime comprising busulfan, fludarabine and ATG. The patient developed temporary graft versus host disease of the skin and intestine but suffered no other severe transplant-related side effects; donor chimerism was not reported in this study. ${ }^{3}$ Shah et al used a 9/10 matched unrelated donor and the same chemotherapy regimen to transplant their patient (Patient 7 ) who engrafted at day +28 . Despite successful initial engraftments, she was again G-CSF- and transfusion-dependent by day +60 in the context of donor chimerism at $81 \%$ and a prolonged rhinovirus infection. An unconditioned stem cell boost together with tacrolimus on day +119 rescued this patient's graft and at day +150 she was reported to have a normal neutrophil count and to be transfusion independent. ${ }^{6}$

At our center, we have transplanted five patients with mutations in the VPS45 gene with one death (detailed above and in Table 1), and four surviving patients. We will now detail the progress of the four surviving patients, two of whom have been previously reported and two previously unreported cases. All patients in our cohort are young Palestinian children born to consanguineous parents with the c.671 C>A; p.T224A mutation in the VPS45 gene.

Of our two previously reported patients, one patient (Patient 1,) was transplanted at eight months of age using fludarabine, busulfan, thiotepa and ATG from a matched sibling donor, who is a carrier of the VPS45 gene mutation. He developed a pervasive development disorder for unknown reasons following his transplant, but is now more than seven years post-transplant and has never displayed graft versus host disease, remains transfusionindependent and demonstrates $100 \%$ donor chimerism. Our other previously reported patient (Table 1, Patient 5) was transplanted at 12 months of age using a bone marrow graft from her grandfather and a conditioning regime of fludarabine, treosulphan, thiotepa and ATG, with $100 \%$ donor chimerism at five months post-transplant. Unfortunately, this child subsequently eight months after the transplant developed steadily decreasing donor chimerism, which failed to improve with multiple donor lymphocyte infusions. In parallel with falling chimerism, severe neutropenia reappeared. The family declined to proceed to a second HSCT and five years after the transplant, she is now six years old, demonstrates $57 \%$ donor chimerism (an improvement from 12\% two years post-transplant) with ANC $0.1 \times$ $10^{9} / \mathrm{L}$. Nevertheless, she is in good clinical condition with a Karnovsky score of 90, no need 
for blood or platelet transfusions, has undertaken her post-transplantation vaccinations, and has remained out of hospital.

We now detail the pre-and post-transplant course of two previously unreported patients who reinforce the clinical phenotype of patients with deficiency of VPS45 protein. Our third successful transplant was a six-month-old female (Patient 8) whose older brother had the same VPS45 mutation and died from infection and bone marrow failure before a suitable donor could be found. Prior to transplant, this infant suffered recurrent pneumonia, skin abscesses, buccal candidiasis, and anaemia and neutropenia. She was transplanted using fludarabine, busulphan, thiotepa and ATG with peripheral blood from a 10/10 matched sibling donor, who is heterozygous for the same VPS45 mutation. Neutrophil engraftment occurred on day 11 , and platelets at day 33 post-transplantation. Her post-transplant course was complicated by hypertension, cutaneous infection with Klebsiella and Pseudomonas, and local Bacillus Calmette-Guerin infection. These complications were successfully treated with anti-hypertensives, meropenem, and a prolonged course of anti-mycobacterial therapy, respectively. She is now 18 months post-transplant with full donor chimerism, is transfusionindependent and has ceased all immunosuppressive medications.

Our fourth successful transplant was a ten-month-old male (patient 9), the first child to consanguineous parents. He had suffered from four episodes of high fever, extremely elevated inflammatory markers (e.g. C-reactive protein $400 \mathrm{mg} / \mathrm{L}$ ) and persistent neutropenia and thrombocytopenia since birth; anemia was present, but mild. He had been treated empirically for meningitis for each of these fever episodes, although no organisms were ever found on cerebrospinal fluid culture. He demonstrated hepatosplenomegaly and nephromegaly on ultrasound scan; he was also failing to thrive with delayed gross motor development, but no other neurological impairement. A c.671 C>A; p.T224A mutation in $V P S 45$ was found on whole exome sequencing and homozygous inheritance from carrier parents was confirmed via Sanger sequencing. A bone marrow biopsy demonstrated increased deposition of reticulin fibres and aspiration yielded a dry tap. He received $10 \times 10^{6}$ CD34+ cells from a 9/10 matched unrelated donor and a conditioning regime that consisted of busulfan, fludarabine, thiotepa and ATG. He is currently day 60 post-transplant and achieved neutrophil engraftment on day 15 . He currently has mild cutaneous acute graft versus host disease and hypertension, which are being managed with immune suppression (prednisolone, mycophenolate, and cyclosporine) and amlodipine, respectively.

Whilst HSCT can cure this disease, some clinical features took some time to resolve, and resolution may well depend on the degree of post-transplantation donor chimerism. Of our successfully transplanted patients, patients 1 and 8 demonstrate resolution of hepatosplenomegaly and nephromegaly, but this took approximately one year to occur; patient 9's hepatosplenomegaly is steadily improving. Patient 5, with $57 \%$ donor chimerism, continues to demonstrate hepatosplenomegaly and nephromegaly and bone marrow biopsy indicates ongoing myelofibrosis. None of our other patients underwent post-HSCT bone marrow biopsy as their cell counts returned to normal and it was not clinically indicated. 


\section{Discussion}

$V P S 45$ gene mutation is a very rare primary immune deficiency, with 19 patients, including those in this report, now described in the literature. ${ }^{3-7}$ Nine bone marrow transplants have now been detailed, with three deaths and six patients surviving with varying degrees of posttransplant disease resolution. Although the numbers are small, in our experience the two most significant impediments to successful transplantation in VPS45 are myelofibrosis and the presence of severe bacterial and fungal infections pre-transplantation. Thus, the first element of our approach to treatment is an early clinical suspicion for this disease. In a consanguineous child with SCN unresponsive to GCSF, and with any evidence of broader bone marrow failure, we move rapidly to genetic sequencing to identify the diagnosis. We also undertake simultaneous human leukocyte antigen (HLA) typing of the nuclear and extended family, and commence an international donor search if no family donor is available. Any infections must be treated aggressively with anti-microbial therapy, with involvement of the infectious diseases team. Pseudomonal, gram-positive, and fungal infections may be deep-seated so blood and tissue cultures should be combined with appropriate imaging to locate any locus of infection. Should a patient present prior to the development of such infections, we recommend prophylactic anti-microbials, including Pneumocystis jirovecii prophylaxis with trimethoprim/sulfamethoxazole, as well as an antifungal drug ( e.g. voriconazole).

In preparing the patient for transplantation, supportive care is vital. In particular supporting the patient's bone marrow failure with transfusion of blood and platelets as needed, and ensuring adequate nutritional support in light of the severe failure to thrive that can occur with this disease, so dietician input is recommended. These measures, combining early genetic diagnosis, transplant donor identification, treatment of infections, and vigorous supportive care will ensure the patient is as healthy as possible prior to transplant with minimal end-organ damage. We do not undertake disease specific organ evaluation, such as an MRI brain, unless it is clinically indicated. Because of the eventual post-HSCT resolution of splenomegaly as evidenced by our patients with $100 \%$ donor chimerism, we do not recommend pre-HSCT splenectomy or radiation.

For transplantation itself, we recommend undertaking the procedure as early as possible as a matter of urgency, using as closely matched a donor as possible (sibling, family, or unrelated), and employing a myeloablative conditioning regime using busulfan.

Heterozygous HLA-matched family donors appear to be a safe option for transplant as patients 1 and 8 were transplanted from carrier donors and are now disease-free. Because VPS45 leads to a diseased bone marrow niche, a myeloablative regime ensures as through a clearance as possible of any diseased blood cells that would otherwise occupy marrow space and continue to secrete bone marrow-damaging cytokines. This is in contrast to HSCT for adult-onset PMF, where a reduced-intensity fludarabine-based regime appears to provide reduced morbidity and increased disease-free survival, although further studies are required. ${ }^{7}$ We have not detected any short- or long-term toxicity in our three patients who have undergone myeloablative conditioning regimes, and only patient 9 developed mild cutaneous GvHD which resolved with prompt steroid therapy. Of course, longer follow-up is required to detect any post-chemotherapy complications. 
Of the two HSCTs we have performed from bone marrow-derived stem cells, one recipient is now disease-free and the other has mixed donor chimerism, but both achieved neutrophil engraftment approximately one week later than our patients transplanted using a peripheral blood stem cell graft. Thus, to achieve earlier and more sustained engraftment, peripherally sourced hematopoietic stem cells may be superior. Our only patient transplanted from umbilical cord blood with a treosulfan-based conditioning regime failed to engraft and died 31 days post-transplantation suggesting that hematopoietic stem cells from cord blood struggle to engraft in the recipient's hostile bone marrow niche. Thus, we do not recommend cord blood HSCT for VPS45.

As detailed in a number of the published transplants, falling chimerism may pose difficulty, again likely secondary to the diseased bone marrow niche. We recommend aiming for full donor chimerism (at our center we can assess only whole blood chimerism), as this appears to offer the best chance of engraftment and cure. As such, transplant teams should tailor their immunosuppressive regimes appropriately and prepare patients for the possible need for donor stem cells top up; we do not use post-transplant G-CSF as a matter of protocol, although this could be trialled if clinically indicated. Despite this recommendation for full donor chimerism, patient 5 demonstrates the possibility of survival with mixed chimerism and the presence of some ongoing disease. Donor Lymphocyte Infusion (DLI) in this case failed to raise the level of donor chimerism, possible because it was not performed until eight months post-HSCT, when the bone marrow niche was replete with cells and the new cells were unable to engraft; earlier DLI may be more successful.

In summary, VPS45 gene mutation is a rare and severe primary immune deficiency that requires early clinical suspicion and aggressive treatment if premature death secondary to bone marrow failure and overwhelming infection is to be avoided. This disease offers important insights into the impact of impaired protein trafficking on the hematopoiesis and the bone marrow itself, with implications for transplantation of other diseases leading to myelofibrosis. There remains much to learn about the molecular and cellular mechanisms linking the genetic defect to the clinical phenotype, and we hope that as more children are diagnosed, translationally investigated, and successfully transplanted, these mechanisms will be uncovered. Several reported patients have demonstrated non-hematological manifestations of disease (developmental delay, dysmorphic features), and continued vigilance of such potential complications and investigation of their connection to VPS45 protein is required. Our center has transplanted five of the nine HSCTs reported in children with confirmed VPS45 mutation; we hope that by sharing our approach to treatment, additional successful transplants will be undertaken at other centers, and a consensus approach will eventually become possible.

\section{Acknowledgments}

We thank Dr Lisa Madden for sharing unpublished data regarding her patient. We would like to thank our patients and their families, as well as departmental nursing and administrative staff for their tireless commitment to patient care. We would also like to thank Professor Zeev Rotstein, Director of The Hadassah Medical Center for his support of the department and the patients.

Funding Information 
Bella Shadur's position is supported by the Australian Government Research Training Program Scholarship. This work was supported by the Deutsche Forschungsgemeinschaft (Discovery and Evaluation of new Combined Immunodeficiency Disease Entities; grant DFG WA 1597/4-2) and the ERA-Net ERARE consortium EURO-CID. Peter Newburger is supported by the following grants: NIH R24 AI049393 and US Department of Defense award W81XWH-17-1-0110.

\section{Abbreviations}

$\begin{array}{ll}\text { ATG } & \text { Anti-Thymocyte Globulin } \\ \text { BCG } & \text { Bacillus Calmette-Guerin } \\ \text { BM } & \text { Bone marrow } \\ \text { DLI } & \text { Donor Lymphocyte Infusion } \\ \text { G-CSF } & \text { Granulocyte colony stimulating factor } \\ \text { HLA } & \text { Human Lymphocyte Antigen } \\ \text { HSCT } & \text { Hematopoietic stem cell transplantation } \\ \text { MRD } & \text { Matched related donor } \\ \text { MSD } & \text { Matched sibling donor } \\ \text { MUD } & \text { Matched unrelated donor } \\ \text { PMF } & \text { Primary myelofibrosis } \\ \text { SCN } & \text { Severe congenital neutropenia } \\ \text { TGF- } \beta & \text { Transforming Growth Factor } \beta \\ \text { UCB } & \text { Umbilical cord blood } \\ \text { VPS45 } & \text { Vacuolar protein sorting protein } 45\end{array}$

\section{References}

1. Mitton B, de Oliveira S, Pullarkat S, Moore T. Stem Cell Transplantation in Primary Myelofibrosis of Childhood. J Pediatr Hematol Oncol 2013;35(3):e120-e2. [PubMed: 23511496]

2. DeLario M, Sheehan A, R A, Bertuch A, Vega C, Webb C, et al. Clinical, Histopathologic, and Genetic Features of Pediatric Primary Myelofibrosis- An Entity Different from Adults. Am J Hematol 2012;87(461-464).

3. Meerschaut I, Bordon V, Dhooge C, Delbeke P, Vanlander A, Simon A, et al. Severe Congenital Neutropenia with Neurological Impairment Due to a Homozygous VPS45 p.E238K Mutation: A Case Report Suggesting a Genotype-Phenotype Correlation. Am J Med Genet Part A 2015;167A: 3214-8. [PubMed: 26358756]

4. Vilboux T, Lev A, Malicdan M, Simon A, Jarvinen P, Racek T, et al. A Congenital Neutrophil Defect Syndrome Associated with Mutations in VPS45. NEJM 2013;369(1):54-65. [PubMed: 23738510]

5. Stepensky P, Saada A, Cowan M, Tabib A, Fischer U, Berkun Y, et al. The Thr224Asn Mutation in the VPS45 Gene is Associated with the Congenital Neutropenia and Primary Myelofibrosis of Infancy. Blood 2013;121(25):5078-87. [PubMed: 23599270] 
6. Shah R, Munson M, Wierenga K, Pokala H, Newburger P, Crawford D. A Novel Homozygous VPS45 p.P468L Mutation Leading to Severe Congenital Neutropenia with Myelofibrosis. Pediatr Blood Cancer 2017;64(e26571).

7. Stepensky P, Simanovsky N, Averbuch D, Gross M, Yanir A, Mevorach D, et al. VPS45-Associated Primary Infantile Myelofibrosis - Successful Treatment with Hematopoietic Stem Cell Transplantation. Pediatr Transplantation 2013;17:820-5.

8. Bryant N, James D. VPS45p Stabilizes the Syntaxin Homologue TIg2p and Positively Regulates SNARE Complex Formation. The EMBO Journal 2001;20(13):3380-8. [PubMed: 11432826]

9. Toonen R, Verhage M. Vesicle Trafficking: Pleasure and Pain from SM Genes. TRENDS in Cell Biology 2003;13(4):177-86. [PubMed: 12667755]

10. Gengyo-Ando K, Kuroyanagi H, Kobayashi T, Murate M, Fujimoto K, Okabe S, et al. The SM Protein VPS-45 is Required for RAB-5-Dependent Endocytic Transport in Caenorhabditis elegans. EMBO Reports 2007;8:152-7. [PubMed: 17235359]

11. Rahajeng J, Caplan S, Naslavsky N. Common and Distinct Roles for the Binding Partners Rabenosyn-5 and VPS45 in the Regulatory of Endocytic Traffickin in Mammalian Cells. Exp Cell Res 2010;316(5):859-74. [PubMed: 19931244]

12. Skokowa J, Dale D, Touw I, Zeidler C, Welte K. Severe Congenital Neutropenias. Nat Rev Dis Primers 2017;3.

13. Glaubach T, Minella A, Corey S. Cellular Stress Pathways in Pediatric Bone Marrow Failure Syndromes: Many Roads to Lead to Neutropenia. Pediatr Res 2014;75(1):189-95. [PubMed: 24192702]

14. Guerrero JA, Bennett C, van der Weyden L, McKinney H, Chin M, Nurden P, et al. Gray Platelet Syndrome: Proinflammatory Megakaryocytes and Alpha-granule Loss Cause Myelofibrosis and Confer Metastasis Resistance in Mice. Blood 2014;124(24):3624-35 [PubMed: 25258341] 


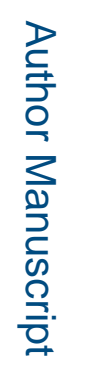

로을

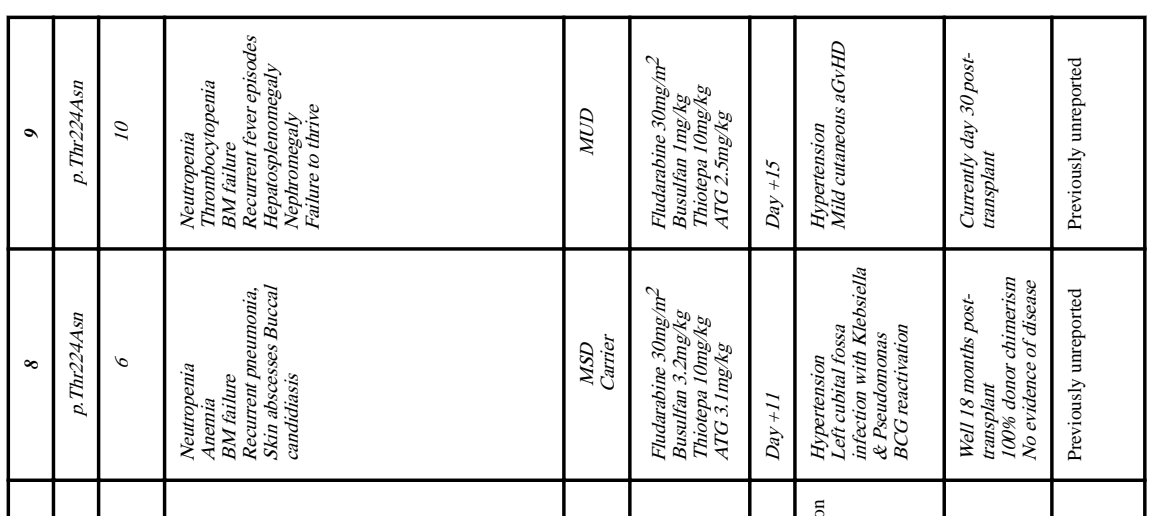

?

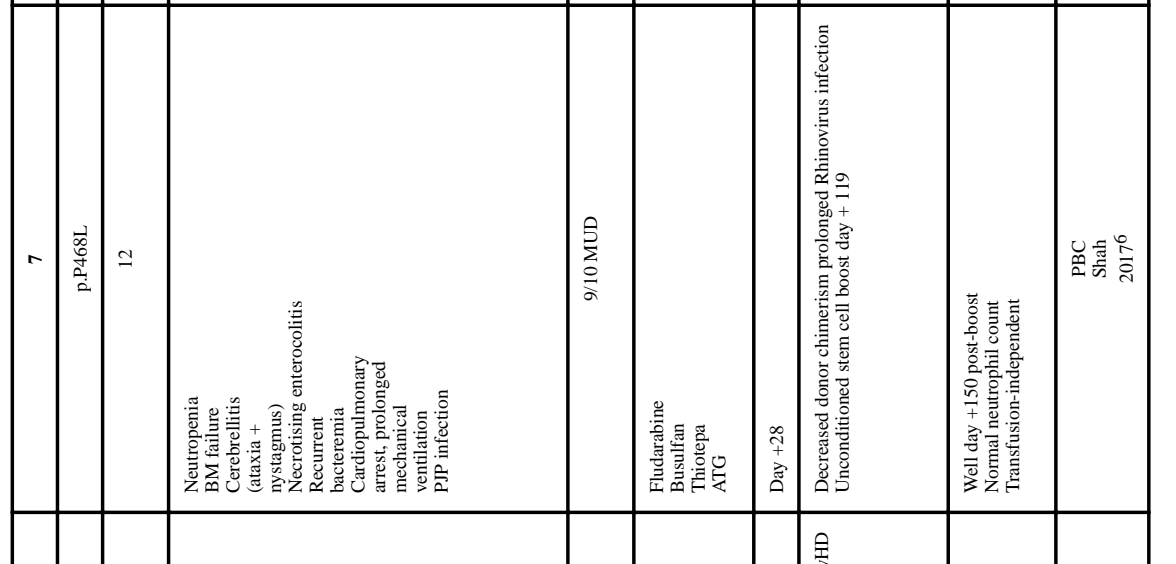

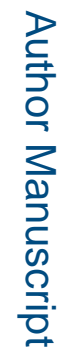



Pediatr Blood Cancer. Author manuscript; available in PMC 2020 January 01. 